\title{
RÉPARTITION SPATIALE ET PRÉFÉRENCES TROPHIQUES de Glossina palpalis Palpalis DANS LE FOYER FORESTIER DE ZOUKOUgBeu (COTE D'IVOIRE). IMPLICATIONS ÉPIDÉMIOLOGIQUES
}

\author{
SANÉ B.*, LAVEISSIÈRE C.*** MÉDA H.A.***
}

Summary : SPATIAL DISTRIBUTION AND BLOODMEAL PREFERENCES OF GLOSSINA PALPALIS PALPALIS IN THE FOREST FOCUS OF ZOUKOUGBEU. EPIDEMIOLOGICAL CONSEQUENCES

In the sleeping sickness focus of Zoukougbeu (Côte d'lvoire), in the cropping areas which are favourable for disease transmission, more than a quarter of the flies collected were found to have fed on domestic pigs. The sites where Glossina palpalis palpalis was caught fed on these animals were concordant with the sites where the patients were present. These results might indicate that in Zoukougbeu, but perhaps also in other sleeping sickness foci, the pig could play an active role in disease transmission, allowing the parasite to spread widely via the tsetse.

KEY WORDS : Human African Trypanosomosis, Glossina, trophic preference, reservoir.

\section{Résumé :}

Dans le foyer de maladie du sommeil de Zoukougbeu /Côte d'Ivoirel, plus d'un quart des glossines capturées s'étaient gorgées sur cochon en zone de plantations, lieux préférentiels de transmission de la trypanosomose humaine. En outre, on y observe une concordance nette entre la localisation des sites où Glossina palpalis palpalis a été capturée gorgée sur ces animaux et la présence de trypanosomés. Ceci laisse penser que, dans le foyer de Zoukougbeu, mais peutêtre aussi dans d'autres foyers de trypanosomose humaine africaine du secteur forestier, le cochon joue un rôle plus actif qu'on ne le pense généralement, dans la transmission de l'endémie en permettant une large diffusion du parasite par l'intermédiaire des glossines.

MOTS CLÉS : Trypanosomose humaine africaine, Glossina, préférences trophiques, réservoirs

de la Côte, le guib harnaché (Tragelaphus scriptus) a constitué la source de nourriture la plus importante des glossines en zones de plantations (Sané et al., 2000). Les inter-relations étroites entre l'homme, la glossine et cette antilope réservoir de T. $b$. gambiense (Molyneux, 1973) ont été capitales sur le plan épidémiologique (Laveissière et al., 1985). Dans la zone de la présente étude, le foyer de Zoukougbeu, le cochon, également réservoir potentiel de cette espèce de trypanosome, a été avec l'homme, le mammifère le plus sollicité par les glossines dans les plantations (Sané et al., 2000). L'analyse de la diversité alimentaire du vecteur dans différents foyers a permis de montrer que l'alternance de repas pris sur homme et sur animaux favorise la transmission de la maladie du sommeil (Sané et al., 2000). Elle a montré que, dans les foyers forestiers étudiés en Côte d'Ivoire, le foyer de Zoukougbeu a été le seul où un important pourcentage de repas a été pris sur cochon en zone de plantations. L'originalité de ce travail consiste à analyser plus précisément, dans un cadre spatial donné, la distribution des malades en relation avec certaines préférences trophiques de G.p.palpalis, pour rechercher le (ou les) réservoirs incriminés dans la transmission de la maladie du sommeil. La connaissance de ces réservoirs dans le schéma épidémiologique de la THA peut contribuer à rendre plus efficace la lutte contre l'endémie som-

meilleuse.
* Institut Pierre Richet, Organisation de Coordination et de Coopération pour la lutte contre les Grandes Endémies (OCCGE), BP 1500, Bouaké 01, Côte d'Ivoire.

** Organisation de Coordination pour la lutte contre les Endémies en Afrique Centrale (OCEAC), BP 288, Yaoundé, Cameroun.

*** Projet Sida 2 Bénin, 08 BP 900 Tri Postal Cotonou, Bénin Correspondance : Dr Sané Bocar.

Tél : (225) 31633746 - Fax : (225) 31632738. 


\section{MATÉRIELS ET MÉTHODES}

I e foyer de Zoukougbeu est localisé au centreouest de la Côte d'Ivoire. Les villages de ce foyer sont en majorité situés non loin des bas-fonds humides dont certains traversent de nombreuses plantations. On pratique, dans la majorité de ces villages, des élevages de cochons. Ces animaux sont laissés en divagation dans et autour des agglomérations.

Les enquêtes ont été réalisées en 1993. Les captures de Glossina p. palpalis ont été faites, durant quatre jours consécutifs, à l'aide de pièges "Vavoua" (Laveissière \& Grébaut, 1990). Ces pièges ont été placés en zone de plantations éloignée des villages et en lisière de village. Les glossines, mâles et femelles, capturées ont toutes été disséquées. Les intestins contenant un repas de sang, quel que soit leur état de digestion, ont été prélevés. Ils ont été étalés sur un disque de papier chromatographique (Whatman $n^{\circ} 1$ ). Les repas de sang contenus dans les intestins ont été analysés par la technique de fixation du complément à l'Institüt für Veterinärmedizin de Berlin (Staak et al., 1986).

Le test du $\chi^{2}$ de Pearson a été utilisé, avec un risque de première espèce de $5 \%$, pour comparer les proportions de repas pris sur les différents hôtes.

\section{RÉSULTATS}

L a répartition des repas pris par G. p. palpalis dans les plantations et en lisières de village est illustrée par la figure 1.

Les pourcentages de repas pris sur cochon ont été significativement plus importants en lisières de village que dans les plantations $\left(\chi^{2}=31,70 ; \mathrm{ddl}=1 ; \mathrm{p}<0,001\right)$. Au village, le cochon représente la principale source de nourriture des glossines capturées.

En zone de plantations, les glossines se gorgent préférentiellement sur l'homme (près de $47 \%$ des repas identifiés), ensuite sur le cochon (25\%); les autres hôtes nourriciers (bovidés, reptiles et oiseaux) ont été

\begin{tabular}{lcr}
\hline & \multicolumn{2}{c}{$\begin{array}{c}\text { Glossines gorgées } \\
\text { sur cochons }\end{array}$} \\
\cline { 2 - 3 } & Au moins une & Aucune \\
\hline Nombre de points de capture & 349 & 1903 \\
Sites avec au moins un malade & 84 & 238 \\
$\%$ sites avec au moins un malade & 24,07 & 12,51 \\
\hline
\end{tabular}

Tableau I. - Relation entre les points de capture de G. p. palpalis gorgées sur cochons et l'habitat des malades de THA.

dans l'ensemble peu sollicités (28,1\%). Dans cette zone, 40,2 \% de sites de captures des glossines gorgées sur cochon ont été situés le long des bas-fonds qui passent à proximité des villages.

La répartition spatiale des sites de captures des glossines gorgées sur cochon semble montrer l'existence d'une relation avec la présence de malades. Cette relation est exprimée dans le tableau I. En effet, parmi les 322 sites fréquentés par au moins un malade (malade travaillant et vivant temporairement ou en permanence dans ce site), le nombre de sites où on a capturé au moins une glossine gorgée sur cochons (24\%) est significativement plus important que ceux où les glossines se sont gorgées sur d'autres vertébrés $(12,5 \%)$ $\left(\chi^{2}=32,14 ; \mathrm{ddl}=1 ; \mathrm{p}<0,0001\right)$.

\section{DISCUSSION-CONCLUSION}

\begin{abstract}
Tette étude souligne une fois de plus le rôle important du cochon comme hôte nourricier de G.p.palpalis dans le foyer de Zoukougbeu. Elle laisse aussi apparaître des différences dans la répartition spatiale des préférences trophiques du vecteur. À titre de comparaison, dans les plantations des foyers de Vavoua et Daniafla (situés aussi au centre-ouest de la Côte d'Ivoire), les pourcentages de repas pris sur cochon ont été respectivement de 7,3\% et de 3,9\% (Laveissière et al., 1985). Ces pourcentages ne diffèrent pas entre eux $(p>0,50)$ mais sont significativement plus faibles que ceux observés à Zoukougbeu $(\mathrm{p}<0,001)$.
\end{abstract}

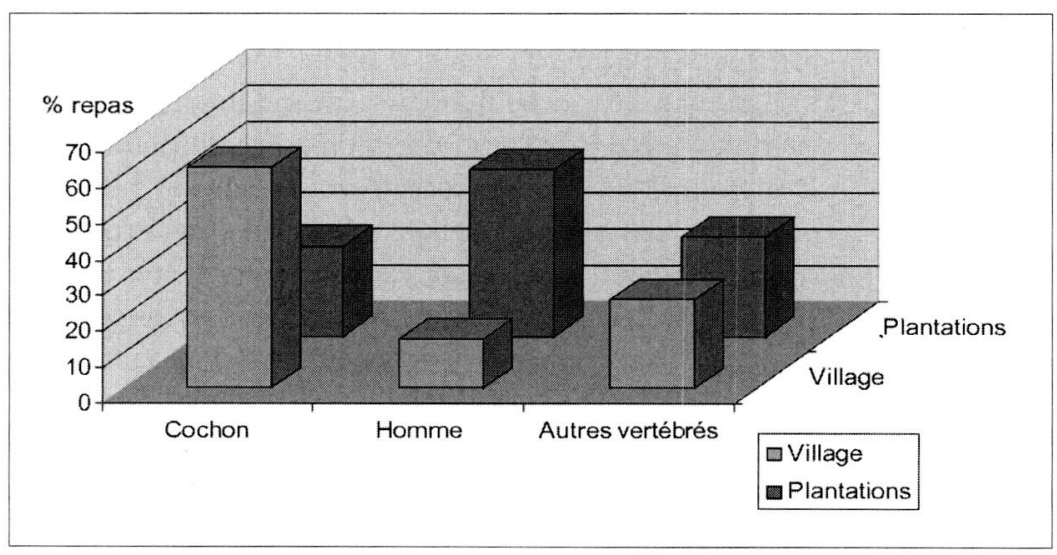

Fig. 1. - Répartition des repas pris par G. p. palpalis dans le foyer de Zoukougbeu. 
En lisière de villages, les pourcentages de repas pris sur ce même hôte à Daniafla $(83,3 \%)$ ne diffèrent pas de ceux de Zoukougbeu ( $p>0,10)$. Mais, dans ce même milieu, le cochon a été plus sollicité à Vavoua $(97,9 \%)$ que dans les deux autres foyers $(\mathrm{p}<0,001)$. Les différences de comportement alimentaire de $G . p$. palpalis observées d'une zone à une autre sont liées à la disponibilité des hôtes, à l'environnement et aux conditions climatiques (Gouteux et al., 1982c; Laveissière et al., 1985).

A Zoukougbeu, contrairement à Vavoua et à Dania (Laveissière et al., 1985), les villages où sont pratiqués des élevages de cochons jouxtent les bas-fonds humides. Les conditions climatiques qui règnent dans ces bas-fonds favorisent le regroupement des cochons laissés en liberté. Ces biotopes sont aussi des lieux de refuge pour les glossines (Laveissière \& Hervouët, 1981; Gouteux et al., 1982b). La disponibilité du cochon dans les gîtes à glossines pourrait expliquer le niveau de contact entre les glossines et cet hôte nourricier dans les bas-fonds proches des villages.

A Vavoua et à Daniafla, les glossines se gorgent sur les cochons en lisière de village. Ces glossines paraissent peu enclines à quitter ce biotope (Gouteux et al., 1982a). La dispersion des glossines en zone forestière est généralement de type centripète (Gouteux et al., 1982a), de la plantation vers le village, souvent en suivant un homme ou un animal. La dispersion centrifuge, des villages vers les plantations et les bas-fonds est, selon les auteurs précédents, toujours de faible amplitude voir nulle puisque les glossines trouvent aux alentours des villages tous les éléments nécessaires à leur survie.

Mais dans le foyer de Zoukougbeu, de nombreuses glossines gorgées sur cet animal ont été capturées loin des villages, dans ou non loin d'un bas-fond humide. Ces glossines une fois gorgées sur cochon dans les basfonds à proximité des villages, se disperseraient, sous l'effet d'un stimulus mal connu, à la recherche d'un lieu de repos, d'un gîte de larviposition ou d'un partenaire sexuel et atteindraient certains campements et basfonds éloignés des villages.

Il semble donc que dans le foyer de Zoukougbeu, et peut être dans d'autres régions forestières, il existe, comme le long des galeries forestières des savanes (Cuisance et al., 1985), une dispersion longitudinale, de forte amplitude, le long des bas-fonds où les facteurs biotiques (hôtes nourriciers, partenaires sexuels...) et abiotiques (température, humidité...) favorables assurent partout la survie de ces glossines.

L'analyse du comportement dispersif et alimentaire du vecteur pourrait contribuer à la compréhension du mécanisme de transmission de la maladie dans les foyers. Dans les plantations, l'hôte préférentiel, à part l'homme, a été le cochon à Zoukougbeu, (25,2\% de repas identifiés) et le guib harnaché à Vavoua, (42,2\% de repas identifiés). Les repas pris sur cette antilope ont été de $1,1 \%$ à Zoukougbeu et 6,8\% à Daniafla. Il est intéressant de constater que, contrairement à Daniafla où la prévalence de la THA était quasi nulle (Laveissière et al., 1985), le cochon à Zoukougbeu et le guib à Vavoua ont été abondamment sollicités par les glossines dans les plantations, lieux préférentiels de transmission de la trypanosomose. Puisque le cochon, (comme le guib), est réservoir potentiel de T. b. gambiense (Molyneux, 1973; Mehlitz, 1985; Mehlitz et al., 1985; Herder et al., 2000), le nombre important de glossines gorgées sur cet animal, dans les sites où vivent des malades de THA, laisse entrevoir l'existence d'une relation entre ces glossines et la transmission de la THA dans ce foyer. Les glossines établies en lisière de villages et dans les bas-fonds proches des villages de Zoukougbeu, pourraient jouer un rôle important dans la transmission de la maladie du sommeil. Ceci permettrait au cochon de devenir, dans le schéma épidémiologique de l'endémie sommeilleuse, un acteur beaucoup plus important qu'il n'a été considéré jusqu'à maintenant. Il ne serait plus un réservoir "passif" faisant écran entre la glossine et l'homme (Laveissière \& Challier, 1976 ; Mehlitz, 1977; Gibson et al., 1978; Baldry, 1980) mais, dans certaines conditions, telles celles qui prévaleraient à Zoukougbeu, un réservoir "actif" favorisant une large diffusion du parasite par les glossines. Il resterait à montrer que $T$. $b$. gambiense qui se développe chez le cochon peut devenir infectant chez la glossine et infecter l'homme.

Il a déjà été rapporté que le cochon et le guib harnaché sont de bons réservoirs de T. $b$. gambiense en Côte d'Ivoire et dans d'autres pays d'Afrique (Molyneux, 1973; Mehlitz, 1985; Mehlitz et al., 1985; Herder et al., 2000). Leur rôle dans le maintien et la diffusion du parasite est de toute évidence lié aux caractéristiques du foyer comme cette étude vient de le montrer. Aujourd'hui, pour affiner les connaissances, pour quantifier l'importance de ce réservoir animal, localiser les sites épidémiologiquement dangereux, il est nécessaire d'utiliser les nouvelles possibilités qu'offre la biologie moléculaire dans la mise au point d'outils performants pour l'identification sub-spécifique des trypanosomes (Herder et al., 2000; Biteau et al., 2000). À terme, ces recherches permettraient certainement d'améliorer l'efficience des campagnes de lutte antivectorielle mais aussi de prendre les mesures qui s'imposent pour contrôler le réservoir animal.

\section{REMERCIEMENTS}

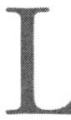
es auteurs tiennent à remercier les Docteurs A. Garcia et P. Solano de l'IPR/IRD Bouaké, ainsi 1 que deux referees anonymes pour la relecture critique de ce manuscrit qui a permis son amélioration. 


\section{RÉFÉRENCES}

BALDRY D.A.T. Local distribution and ecology of Glossina palpalis and $G$. tachinoides in forest foci of West African Human Trypanosomiasis, with special reference to associations between peri-domestic tsetse and their hosts. Insect Sciences Applied, 1980, 1, 85-93.

Biteau N., Bringaud F., Gibson W.C., Truc P. \& Baltz T. Characterization of Trypazoon isolates using a repeated coding sequence and microsatellite markers. Molecular Biochemical Parasitology, 2000, 105, 185-201.

Challier A. \& Gouteux J.P. Ecology and epidemiological importance of Glossina palpalis in the Ivory Coast forest zone. Insect Sciences Applied, 1980, 1, 77-83

Cuisance D. Glossines et trypanosomes. Études de synthèses de CIRAD-EMVT, Montpellier, 1993, 57 pp.

Cuisance D., Février J. \& Filledier J. Dispersion linéaire de Glossina palpalis gambiensis et de Glossina tachinoides dans une galerie forestière en zone soudano-guinéenne (Burkina-Faso). Revue Elevage et Médecine Vétérinaire Pays tropicaux, 1985, 38 (2), 153-172.

Gibson W.C., Mehlitz D., Lanham S. \& Godfrey D.G. The identification of Trypanosoma brucei gambiense in liberian pigs and dogs by resistance to human plasma. Tropenmedizin Parasitologie, 1978, 29, 335-345.

Gouteux J.P., Dongo P. \& Coulibaly D. Observations sur les glossines d'un foyer forestier de trypanosomiase humaine en Côte d'Ivoire. 3. Dispersion et distribution des populations autour d'un village. Tropenmedizin Parasitologie, 1982a, 33, 119-128.

Gouteux J.P. \& Laveissière C. Écologie des glossines en secteur préforestier de Côte d'Ivoire. 4. Dynamique de l'écodistribution en terroir villageois. Cahiers ORSTOM, série Entomologie médicale et Parasitologie, 1982b, 20 (3), 199229.

Gouteux J.P., Laveissière C. \& Boreham P.F.L. Écologie des glossines en secteur pré-forestier de Côte d'Ivoire. 2. Les préférences trophiques de Glossina palpalis s.l.. Cahiers ORSTOM, série Entomologie médicale et Parasitologie, 1982c, 20, 3-18

Herder S., Njiokou F., Mgbedie M., Simo G., Nkinin S., Kohagne L. \& Laveissière C. Existe-t-il un réservoir animal "sauvage" de la trypanosomose humaine africaine au sud Cameroun? Congrès de la société française de parasitologie, 1-3 mars 2000.

LAVEISSIÈre C. \& Challier A. Le foyer de trypanosomoiase humaine de Bouaflé (Côte d'Ivoire) : enquête épidémiologique et entomologique, propositions pour une campagne insecticide. Documentation Technique OCCGE, 1976, 6308 : 20 pp.

Laveissière C., Couret D. \& Hervouët J.P. Localisation et fréquence du contact homme/glossines en secteur forestier de Côte d'Ivoire. 1. Recherche des points épidémiologiquement dangereux dans l'environnement végétal. Cahiers ORSTOM, série Entomologie médicale et Parasitologie, 1986a, 1, 21-35.

Laveissière C., Couret D. \& Hervouët J.P. Localisation et fréquence du contact homme/glossine en secteur forestier de
Côte d'Ivoire. 2. Le facteur humain et la transmission de la trypanosomiase. Cabiers ORSTOM, série Entomologie médicale et Parasitologie, 1986b, 24, 45-57.

Laveissière C., Couret D., StaAk C. \& Hervouët J.P. Glossina palpalis et ses hôtes en secteur forestier de Côte d'Ivoire. Relation avec l'épidémiologie de la Trypanosomiase humaine. Cabiers ORSTOM, série Entomologie médicale et Parasitologie, 1985, 23 (4), 297-303.

LAveissière C. \& GréBAut P. Recherche sur les pièges à glossines (Diptera : Glossinidae). Mise au point d'un modèle économique : le piège "Vavoua". Tropenmedizin Parasitologie, 1990, 41, 185-192.

Laveissière C. \& Hervouët J.P. Populations de glossines et occupation de l'espace. Enquête entomologique préliminaire dans la région de la Lobo (Côte d'Ivoire). Cabiers ORSTOM, série Entomologie médicale et Parasitologie, 1981, 19, 247-260.

MenLitz D. The behaviour in the blood incubation infectivity test of four Trypanozoon strains isolated from pigs in Liberia. Transactions of the Royal Society of Tropical Medicine and Hygiene, 1977, 71, 86.

Mehlitz D. Les réservoirs animaux de la maladie du sommeil. Documentation OMS, TRY/EC/WP/85, 1985, 12 , $128 \mathrm{pp}$

Mehlitz D., Zillmann U. \& SaChs R. Further evidence of animal reservoir host of gambiense sleeping sickness: mixed populations of Trypanosoma brucei gambiense and T. $b$. bruce in naturally infected pigs in the Ivory Coast. ISCTRC, Harare, 4-9 March 1985: 128-129.

Molyneux D.H. Animal reservoirs and gambian trypanosomiasis. Annales de la Société Belge de Médecine Tropicale, 1973, 53, 605-618.

STAAK C., KAMPE U. \& KORKOwSKI G. Species identification of blood-meals from tsetse flies (Glossinidae). Tropenmedizin Parasitologie, 1986, 37, 59-60.

Sané B., Laveissière C. \& MÉda H.A. Diversité du régime alimentaire de Glossina palpalis palpalis en zone forestière de Côte d'Ivoire : relation avec la prévalence de la trypanosomiase humaine africaine. Trop. Med. Int. Hlth, 2000, 5 (1), $73-78$

Reçu le 17 février 2000 Accepté le 5 juillet 2000 